\title{
Numerical Simulation of Near-Field Seismoacoustic Probing of a Layer Inclusion in a Homogeneous Infinite Medium
}

\author{
Yury Mikhailovich Zaslavsky, Vladislav Yuryevich Zaslavsky \\ Institute of Applied Physics, Russian Academy of Sciences, Nizhny Novgorod, Russia \\ Email: zaslav@hydro.appl.sci-nnov.ru
}

Received February 19 ${ }^{\text {th }}, 2010$; revised April 2 ${ }^{\text {nd }}, 2010$; accepted May $3^{\text {rd }}, 2010$.

\begin{abstract}
Spatial distribution of acoustic and elastic waves generated by an elementary vibration source at seismic profiling frequencies in an infinite medium close to a layer inclusion, i.e., an extended layer, is numerically simulated. Point dipole radiation in a homogeneous infinite medium separated by a liquid layer of different medium density or acoustic wave velocity is considered. Transverse elastic SH-waves excited by an oscillating power source in a solid medium also located close to the layer of different propagation velocity than the velocity of the vicinity are analyzed. Formulae for the spatial distribution of the wave field amplitude are derived and computer graphics of field distribution images is presented. Wave reflection, penetration deep into the layer inclusion, and transmittance through it are examined. Results of the analysis can be applied to seismoacoustic probing of geologic environment by the near field of a harmonic vibration source.
\end{abstract}

Keywords: Seismoacoustic Probing, Vibration Source, Acoustic, Transverse Waves, Wave Field Amplitudes, Spatial Distribution, Inhomogeneity

\section{Introduction}

New methods of acoustic remote diagnostics of materials and vibroseismic probing of geologic environment are actively developing now. This research is eventually focused on solving the so-called inverse problems, i.e., problems of inversion or reconstruction of a medium by vibroseismic (acoustic) probing data [1,2]. Although some fundamental results have been achieved in developing the theoretical basis of these methods, the relation between the radiation field configurations at the distances of several tens of wavelengths from a vibration source to the parameters of a layered medium structure is not yet studied thoroughly [3]. The study of this relation is required for optimal solution of this problem; analytical results of the so-called direct problems can be used for this purpose. The existence of this relation was considered in previous papers devoted to the analysis of the near elastic-wave field configuration in a medium with an elementary plane layered structure [4-6]. It is assumed that at the distances of the order of several near-surface layer depths being simultaneously the probing inhomogeneity, the field configuration strongly depends on the geometrical parameters of the layered structure and the acoustic parameters of the medium. This informative relation decays, as the distance between the source and the receivers grows. Thus the problem analyzed in the paper can be formulated as numerical simulation and visualization of the structural features of the near field of a harmonic acoustic (vibration) source located close to a layer inclusion characterized by a jump of wave velocity or density relatively to the analogous parameter of the ambient homogeneous medium. The results of the analysis can be of interest for solving the problem of productive layer probing in entrails of the earth using structural features of near seismoacoustic fields of vibration sources, similarly to "near-field" location of inhomogeneities by pulse signals. If this probing is carried out by means of a vibration source operating in the harmonic vibration mode, precisely field configurations should be considered as characteristic informative features. In this case, the problems solved by probing can be generally formulated as the localization of the nearest boundary of inhomogeneity relatively to the source location, the determination of the characteristic spatial scale of the region occupied by inhomogeneity, the estimation of contrast in densities or wave velocities of media in the region 
of inhomogeneity (layer) relatively to internal and external regions.

Analogous problems are set when determining productive layer features in the bottom marine environment, which are probably present in the characteristics of hydroacoustic signals recorded in shelf probing $[1,2,7,8]$.

To extend the application area of the acoustic probing analysis and generalize it to solid media, we also set forth the results of numerical simulation of the amplitude distribution of elastic transverse $\mathrm{SH}$-waves excited by an oscillating power source located analogously to that in the previous acoustic case, i.e., at some distance from the plane parallel layer inclusion having the thickness of one or several wavelengths. To describe the field of a scalar medium (liquid or gas), one scalar potential is sufficient, while the oscillation source is a point oscillating dipole. To describe elastic $S H$-waves in a solid medium in the two-dimensional formulation, we use one component of the vector potential; a harmonically oscillating power source uniformly distributed along an infinite line parallel to the layer boundary has the same orientation of the momentum and radiates transverse $S H$-waves perpendicular to this line. It is interesting to compare wave patterns of acoustic and elastic-wave fields. First we consider a scalar acoustic field and then results of transverse elastic wave analysis.

\section{Near Acoustic Field of a Point Dipole Located Close to a Layer Inclusion in a Homogeneous Infinite Medium}

The geometry of the problem is shown in Figure 1. Three-dimensional infinite space filled with a liquid or gaseous medium and characterized by the parameters $\rho$ and $C$, i.e., the density and the acoustic wave velocity, is separated by a layer infinite in the $x$ and $y$ directions and enclosed in the limits $h<z<h+H$ in the vertical $Z$ direction; it has the same density $\rho$ as the vicinity and differs only by the sound velocity $c(c \neq C)$. The source $F_{0} \vec{z}^{0} \delta(r) \delta(z) e^{-i \omega t}$ is a point dipole having the power (momentum) $F_{0}$ and the oscillation frequency $\omega$; it is a perturbation in the form of $\delta$-functions of the radial $r$ and axial $\mathrm{Z}$ coordinates, oriented along the vertical axis $\left(\vec{Z}^{0}\right.$ is the corresponding unitary vector), and located at the distance $h$ twice larger than its thickness $H$ relatively to one of the layer boundaries (this value is taken for definiteness of calculations). In Figure 1, the entire space is divided into four artificially isolated zones (numerated by 1, 2, 3, and 4). In these four calculation regions due to axial symmetry,

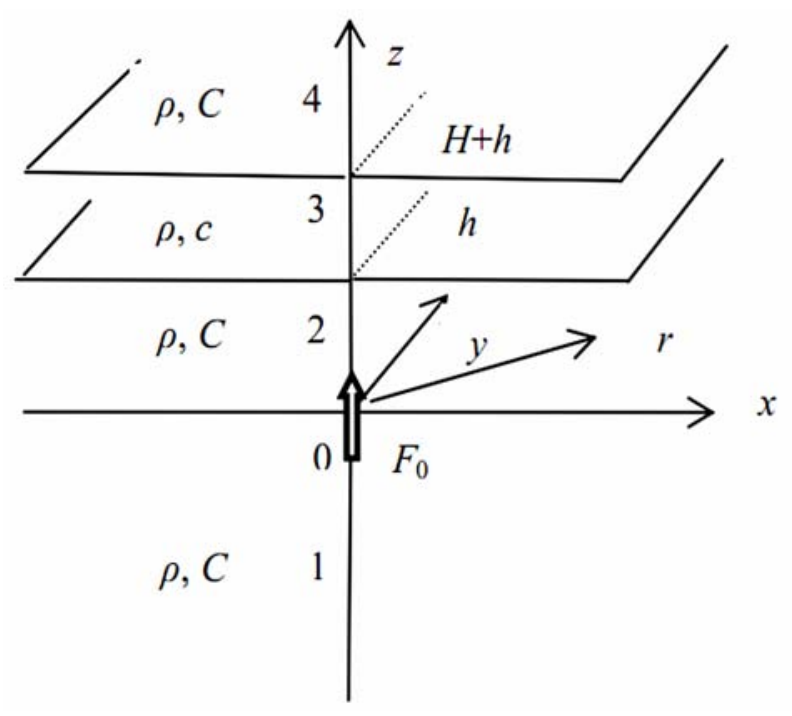

Figure 1. Medium structure and source arrangement for the "scalar" problem

the acoustic shift field can be described by the scalar potential $\varphi$ represented for each of them as Fourier-Bessel integrals, i.e., by the following expressions (the factor $e^{-i \omega t}$ is omitted):

$$
\begin{gathered}
\phi^{(1)}=\int_{0}^{\infty} a(k) e^{-i \mathrm{~K} z} J_{0}(k r) d k, z<0, \\
\phi^{(2)}=\int_{0}^{\infty}\left(b(k) e^{i \mathrm{~K} z}+b^{\prime}(k) e^{-i \mathrm{~K} z}\right) J_{0}(k r) d k, h>z>0, \\
\varphi^{(3)}=\int_{0}^{\infty}\left(B(k) e^{i \kappa z}+B^{\prime}(k) e^{-i k z}\right) J_{0}(k r) d k, \\
H+h>z>h, \\
\varphi^{(4)}=\int_{0}^{\infty} d(k) e^{i K z} J_{0}(k r) d k, z>H+h,
\end{gathered}
$$

where $J_{0}(k r)$ is the zero-order Bessel function, $r$ is the radial coordinate, $k$ is the radial wave number component, i.e., the integration variable, $\mathrm{K}=\sqrt{\omega^{2} / C^{2}-k^{2}}$, $\kappa=\sqrt{\omega^{2} / c^{2}-k^{2}}$, and the indefinite coefficients $a, b, b^{\prime}, B, B^{\prime}, d$ are further calculated from the matching conditions of the $z$-component of the wave displacements $u_{z}$ and the acoustic pressure $p$ at the boundaries of all the four isolated regions.

The problem is based on the solution of a homogeneous acoustic wave equation:

$$
\Delta \varphi^{(1,2,4)}-\frac{1}{C^{2}} \frac{\partial^{2} \varphi^{(1,2,4)}}{\partial t^{2}}=0,
$$




$$
\Delta \varphi^{(3)}-\frac{1}{c^{2}} \frac{\partial^{2} \varphi^{(3)}}{\partial t^{2}}=0 ;
$$

the source operation is written under the appropriate boundary condition instead of being written in the right-hand side of the wave equation:

$$
\begin{aligned}
& p^{(2)}(z=0)-p^{(1)}(z=0)=F_{0} \delta(r) \\
& =\frac{F_{0}}{2 \pi} \int_{0}^{\infty} J_{0}(k r) k d k
\end{aligned}
$$

The relation between the potential $\varphi$, the acoustic pressure $p$, and the $z$-component of the wave displacement $u_{z}$ is commonly known:

$$
p=\rho \omega^{2} \varphi, u_{z}=\nabla_{z} \varphi=\partial \varphi / \partial z
$$

Since the explicit forms of the unknown coefficients are determined, the expressions for acoustic displacements in all spatial regions are written using standard expansions:

$$
\begin{gathered}
u_{z}^{(1)}=\frac{i F_{0}}{4 \pi \rho \omega^{2}} \\
\cdot \int_{0}^{\infty}\left\{1-\left(1+\frac{2 \kappa\left((\mathrm{K}-\kappa) e^{i \kappa H}+(\mathrm{K}+\kappa) e^{-i \kappa H}\right)}{(\mathrm{K}-\kappa)^{2} e^{i \kappa H}-(\mathrm{K}+\kappa)^{2} e^{-i \kappa H}}\right) e^{i 2 K h}\right\}, \\
\cdot e^{-i \mathrm{~K} z} J_{0}(k r) \mathrm{K} k d k \\
u_{z}^{(2)}=\frac{i F_{0}}{4 \pi \rho \omega^{2}} \\
\int_{0}^{\infty}\left\{e^{i \mathrm{~K} z}-e^{i \mathrm{~K}(2 h-z)}\left(1+\frac{2 \kappa\left((\mathrm{K}-\kappa) e^{i \kappa H}+(\mathrm{K}+\kappa) e^{-i \kappa H}\right)}{(\mathrm{K}-\kappa)^{2} e^{i \kappa H}-(\mathrm{K}+\kappa)^{2} e^{-i \kappa H}}\right)\right\} \\
\cdot J_{0}(k r) \mathrm{K} k d k
\end{gathered}
$$

$$
u_{z}^{(3)}=\frac{-i F_{0}}{2 \pi \rho \omega^{2}}
$$

$$
\int_{0}^{\infty} \frac{(\mathrm{K}+\kappa) e^{-i \kappa(H+h-z)}+(\mathrm{K}-\kappa) e^{i \kappa(H+h-z)}}{(\mathrm{K}-\kappa)^{2} e^{i \kappa H}-(\mathrm{K}+\kappa)^{2} e^{-i \kappa H}} e^{i K h} J_{0}(k r) \mathrm{K} \kappa k d k
$$

$$
\begin{aligned}
& u_{z}^{(4)}=\frac{-i F_{0}}{\pi \rho \omega^{2}} \\
& \cdot \int_{0}^{\infty} \frac{e^{i K(z-H)}}{(\mathrm{K}-\kappa)^{2} e^{i \kappa H}-(\mathrm{K}+\kappa)^{2} e^{-i \kappa H}} \\
& \cdot J_{0}(k r) \mathrm{K}^{2} \kappa k d k
\end{aligned}
$$

Specifically, it follows from the latter formula that field $u_{z}^{(4)}$ transmitted through the layer does not depend on the distance $h$ from the source to the layer boundary closest to it. The first and the last formulae describe the acoustic wave field traveling for small distances and also lengths much larger than the wavelength from inhomogeneity and the source. In this case, the integrals in Formulae (5) can be asymptotically estimated, while the wave displacements corresponding to regions 1 and 4 can be given by the expressions:

$$
u_{z}^{(1)}=\frac{F_{0} \cos ^{2} \theta G(\theta)}{4 \pi \rho C^{2} R} e^{i \omega R / C},
$$

In Formula (7), $\Omega=\omega H / c, \quad c^{\prime}=c / C, \chi=H / h$, and the angle $\theta$ is measured from the vertical axis $z$.

The angular characteristics of wave radiation are obtained by Formulae (6) and (7); they show the amplitude angular dependences for the far backscattered wave fields $u_{z}^{(1)}$ and for the fields traveling forward $u_{z}^{(4)}$.

$$
\begin{aligned}
& G(\theta)=1-e^{i \frac{2 \Omega c^{\prime} \cos \theta}{\chi}}- \\
& \frac{\sqrt{1-c^{\prime 2} \sin ^{2} \theta}\left[\left(c^{\prime} \cos \theta-\sqrt{1-c^{\prime 2} \sin ^{2} \theta}\right) e^{i \Omega \sqrt{1-c^{\prime 2} \sin ^{2} \theta}}+\left(c^{\prime} \cos \theta+\sqrt{1-c^{\prime 2} \sin ^{2} \theta}\right) e^{-i \Omega \sqrt{1-c^{\prime 2} \sin ^{2} \theta}}\right]}{\left[\left(c^{\prime} \cos \theta-\sqrt{1-c^{\prime 2} \sin ^{2} \theta}\right)^{2} e^{i \Omega \sqrt{1-c^{\prime 2} \sin ^{2} \theta}}-\left(c^{\prime} \cos \theta+\sqrt{1-c^{\prime 2} \sin ^{2} \theta}\right)^{2} e^{-i \Omega \sqrt{1-c^{\prime 2} \sin ^{2} \theta}}\right]} \\
& \cdot e^{i \frac{2 \Omega c^{\prime} \cos \theta}{\chi}} \\
& u_{z}^{(4)}=\frac{-i F_{0} \Phi(\theta)}{\pi \rho C^{2} c^{\prime} R} e^{-i \omega H \cos \theta / c^{+i \omega R} / C}, \\
& \Phi(\theta)=\frac{\cos ^{3} \theta \sqrt{1-c^{\prime 2} \sin { }^{2} \theta}}{\left[\left(c^{\prime} \cos \theta-\sqrt{1-c^{\prime 2} \sin ^{2} \theta}\right)^{2} e^{i \Omega \sqrt{1-c^{\prime 2} \sin ^{2} \theta}}-\left(c^{\prime} \cos \theta+\sqrt{1-c^{\prime 2} \sin ^{2} \theta}\right)^{2} e^{-i \Omega \sqrt{1-c^{\prime 2} \sin ^{2} \theta}}\right]},
\end{aligned}
$$


The far field characteristics are displayed in Figures 2(a) and (b) (in curves 1,2 , and $3, \Omega=\pi / 2, \pi, 2.5 \pi$ ); these characteristics correspond to waveguide propagation in the layer, i.e., for $c^{\prime}=0.9$. It is seen that in the far zone, the angular pattern of backscattered waves changes as the frequency grows, while the directivity of the field transmitted through the layer remains unchanged and close to the directivity of the dipole source oscillating in a homogeneous infinite medium. The calculation results of antiwaveguide propagation for $c^{\prime}=1.1$ are shown in Figures 3(a) and (b). There is a considerable difference in the angular dependences of the backscattered far field and the field scattered forward. The characteristic of the field traveling forward is the occurrence of sharply directed maxima with simultaneous presence of the central lobe describing the smooth dependence. The backscattered field pattern has only sharply directed maxima analogous to those mentioned above, as applied to the wave field transmitted through the layer, which exist there together with smooth lobes. They, probably, exist due to the so-called nonray waves [3-6].

The spatial distribution of the acoustic field amplitude can be also analyzed by means of numerical calculation of the integrals in Formula (5); in this case, the calculated distances do not exceed the first tens of wavelengths. Since the numerical approach has been employed, the choice of integral signs eliminating ambiguity in the variable $k$ on two-lobe surfaces and the choice of the integration methods essential in the analytical calculations are not discussed.

Now we consider patterns of the spatial amplitude distribution of the $z$-component of wave displacements, which are obtained as a result of numerical simulation using Formula (5) for the same values of acoustic wave velocity jump in the media located inside and outside the layer, i.e., for the waveguide propagation $c^{\prime}=c / C$ $=0.9$ and for the antiwaveguide propagation $c^{\prime}=$ $c / C=1.1$. Note that the actual pattern of the acoustic shift field is to be axially symmetrical relatively to the axis $Z$ and can be a set of interleaved axially symmetrical bodies. However in graphical presentation of the amplitude field distribution, we use the isometric projection, in which the field level is represented as relief rising above the plane $r, z$. The calculated structure of the acoustic displacement field $\left|u_{z}\right|$ is shown in Figures 4(a), 4(b), 4(c) and 4(d) for $c^{\prime}=c / C=0.9$ and in Figures 5(a), 5(b), 5(c) and 5(d) for $c^{\prime}=c / C=1.1$.

Figure 4(a) displays the field fragment corresponding to region 1 located behind the source on the opposite side of the layer region, i.e., for $Z<0$; thus it should be con- sidered turned in the opposite direction along the vertical coordinate $z$ and the corresponding axis in it is denoted $-z$. The same scale is used in both axes. The level decrease is accompanied by the presence of a fan-shaped structure in the field image over the entire plane, which means the oscillating dependence of $\left|u_{z}\right|$ on $r$ for constant $z$ or the oscillating dependence on $Z$ for constant $r$. It follows from the dependence $\left|u_{z}\right|$ on the coordinates that there is an acoustic radiation maximum directed at a small angle to the axis $Z$, which is indicated by "elevation" in the appropriate relief region inclined to this axis. As distinct from Figure 4(a), in Figures 4(b) and 4(c) the scale of the axis $Z$ is 100 times smaller than the scale of the axis $r$. The analyzed spatial interval along the vertical axis amounts to $0 \leq z \leq h$ in Figure 4(b) and to $h \leq z \leq h+H$ in Figure 4(c). In Figure 4(c) for more detailed consideration of the pattern in the radial $r$-direction in the layer region, we used a 10 times smaller scale. In regions 2 and 3 at larger distances from the source, the field amplitude sharply decreases both in radius and vertical $Z$ - direction, which is seen in Figures 4(b) and 4(c). After deep minimum when the face boundary of the layer is approached, sharp decrease of the level is changed by the amplitude growth accompanied by its oscillations. Oscillation amplitude decreases in region 3 are not strong, which indicates that there is the excitation of several interfering modes in the layer; each of the resonance frequencies of these modes being far from the chosen frequency of the source. In region 4 (Figure 4(d)), one can see a comparatively rapid decrease of the field level; it is not so sharp as the level differences in Figures 4(b) and 4(c), if 100-fold scale difference along $Z$ axis in these figures is taken into account. The pattern of the near field in region 4 does not reveal details of the angular concentration of the acoustic field radiated beyond the layer and going to infinity. Therefore, the calculation data obtained from (7) and shown in Figure 3(a) supplement the entire field pattern. At the same time it is evident that even at small distances, the field backscattered by the layer has more peculiarities in its spatial configuration than the field transmitted through the layer outward has in its amplitude distribution. If it is assumed that the spatial configuration can be the informativeness parameter representing the characteristics of the layer itself, then it is seen from comparison that the reflected field contains more information than the field transmitted on the opposite side. In conclusion of this brief review of the wave pattern it can be assumed that amplitude oscillations along the radial and axial coordinates in the near backscattered field is the consequence of interference of the waves reflected from the nearest (face) and the second (external relatively to the source) boundaries. This statement is also applicable to 
other cases considered below, although wave interference in the field transmitted outward is not always so strong.

It follows from Figures 5(a) and 5(b), and $c$ that for $c^{\prime}=c / C=1.1$, the spatial distributions of the wave amplitudes corresponding to spatial regions 1,2 , and 3 have the forms essentially analogous to those considered above. There is an apparent difference from the previous case only in the amplitude distribution in spatial region 4 corresponding to the field transmitted through the layer (Figure 5(d)). In the first case, space-angular oscillations in the transmitted field level were absent; while in considered case, they are present in the three-dimensional image of amplitudes. This is indicated by the fan-shaped angular-periodic structure observed up to some angle to the vertical axis and similar to the structure shown in
Figure 4(a); its angular periodic repetition is approximately the same as in region 1 . The primary role here is, probably, played by nonray waves having a rather high level in the spatial region limited by the sector forming the angle $\gamma= \pm \arccos (C / c)$ with normal to the boundary [3-6].

Thus in the considered cases, there is some difference in the entire pattern of the spatial distribution of acoustic fields, which can be used for remote diagnostics of a probed inhomogeneity. It is evident that spatial amplitude distributions of both the backscattered field and the field transmitted through the layer should be recorded, since the near field structure of the acoustic wave transmitted through inhomogeneity also represents the influence of

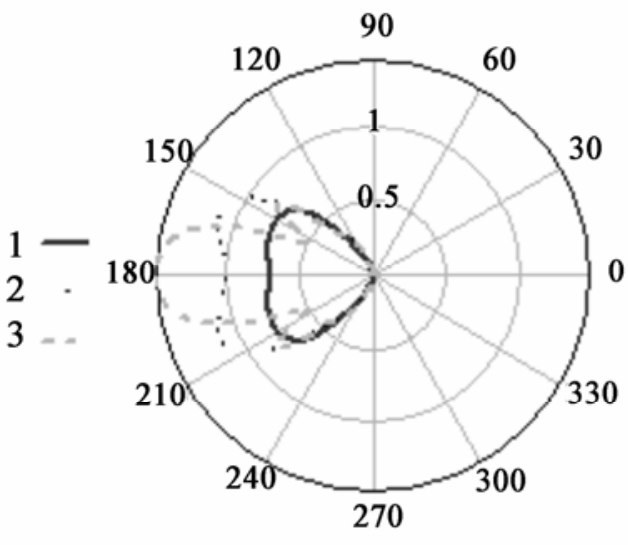

$\Theta$

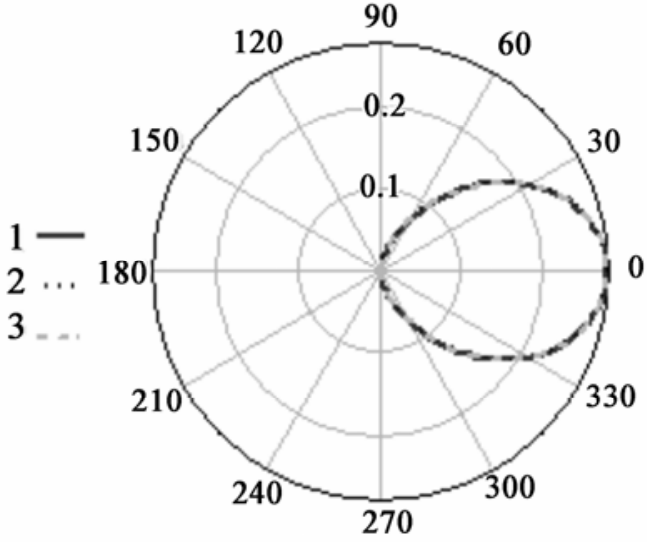

$\Theta$

(a)

(b)

Figure 2. Angular field characteristics (a) $-u_{z}^{(1)},(b)-u_{z}^{(4)}$. Curves 1, 2, and $3-\Omega=\pi / 2, \pi, 2.5 \pi, \quad c^{\prime}=0.9$

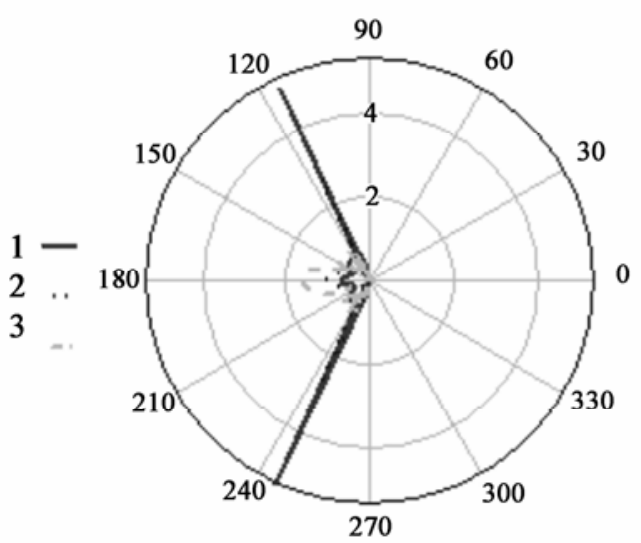

$\Theta$

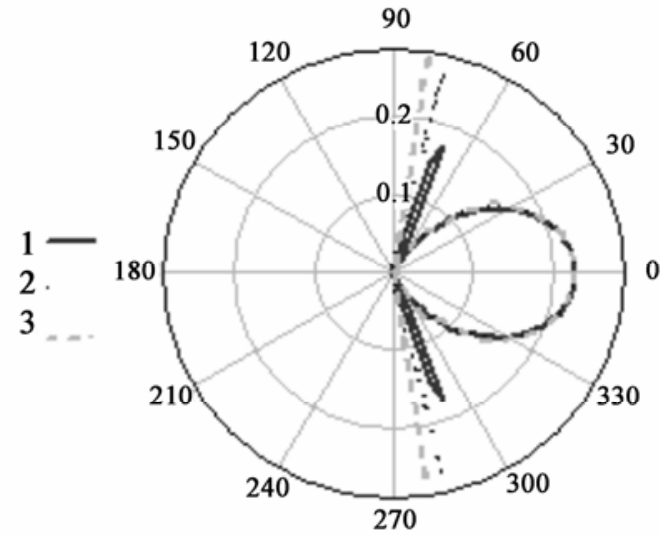

$\Theta$

(a)

(b)

Figure 3. Angular field characteristics: (a) $-u_{z}^{(1)},(\mathrm{b})-u_{z}^{(4)}$. Curves 1,2 , and $3-\Omega=\pi / 2, \pi, 2.5 \pi, \quad c^{\prime}=1.1$ 


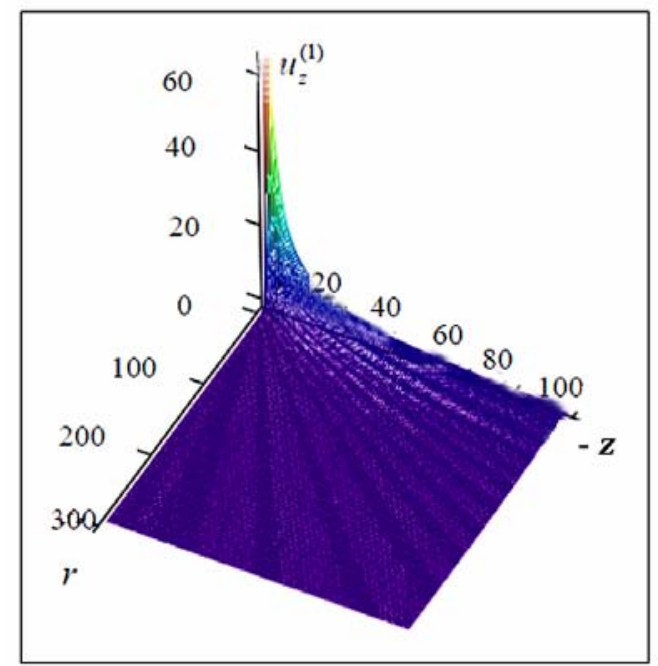

(a)

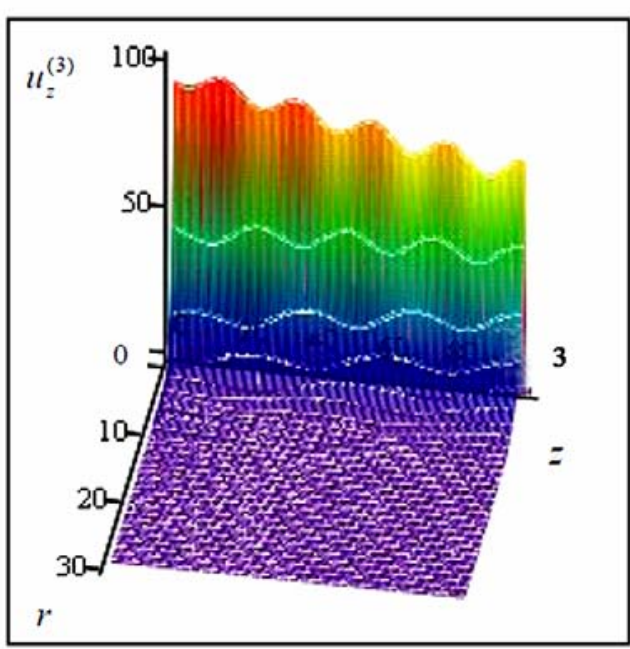

(c)

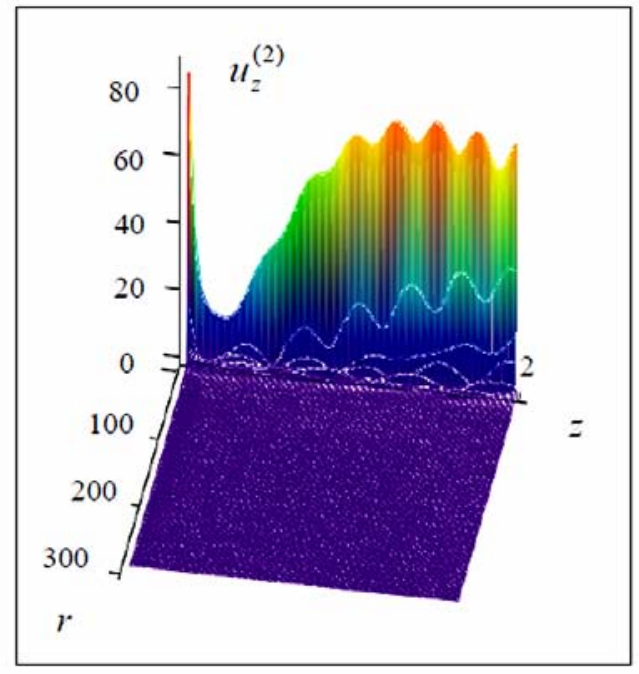

(b)

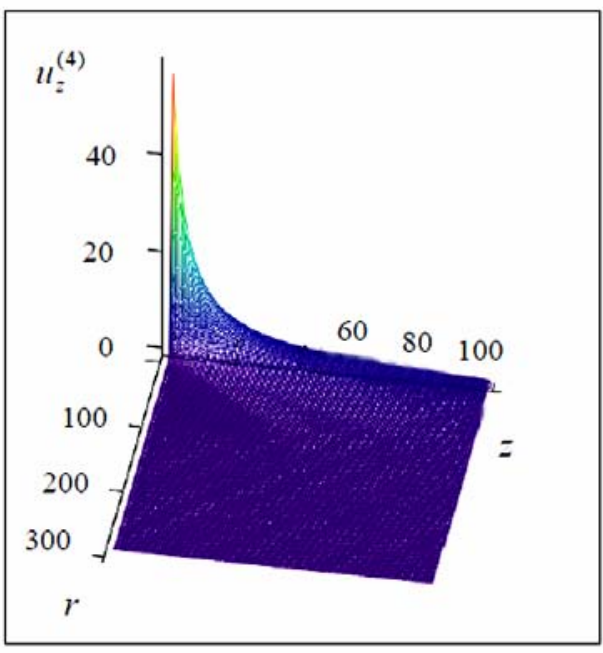

(d)

Figure 4. Fragments of the amplitude distribution pattern of the field $\left|u_{z}\right|$; relief over the coordinate plane $r-z$ : (a) - Region 1; (b) - Region 2; (c)-Region 3; (d) - Region 4. $c^{\prime}=0.9, \omega h / C=11.1 \pi, \omega H / c=5 \pi$

the inhomogeneity parameters.

The problems of the backscattered acoustic field of a dipole harmonic source and of the field transmitted through a layer inclusion into a homogeneous infinite medium (when the media differ only in density) are solved analogously to the stated above. If we consider the same geometry of the layer-medium structure as in the case of Figure 1, use the same arrangement of the source relatively to the boundaries ( $h$ is the distance between the source and the face boundary of the layer and $H$ is the layer thickness), and assume that the sound velocity $C$ is equal everywhere, the density of the medium in the vicinity is $\rho_{1}$, while in the layer is $\rho_{2}$, it is easy to obtain the following expressions for the acoustic displacements in the reflected acoustic field $u_{z}^{(1)}$ and the acoustic field $u_{z}^{(4)}$ transmitted through the layer:

$$
u_{z}^{(1)}=\frac{-i F_{0}}{4 \pi \rho_{1} \omega^{2}} \int_{0}^{\infty}\left(e^{i 2 \mathrm{~K} h}-1-\frac{2\left(\cos (\mathrm{K} H)-i \frac{\rho_{1}}{\rho_{2}} \sin (\mathrm{K} H)\right)}{\left(2 \cos (\mathrm{K} H)-i\left(\frac{\rho_{2}}{\rho_{1}}+\frac{\rho_{1}}{\rho_{2}}\right) \sin (\mathrm{K} H)\right)} e^{i \mathrm{~K}(h+H)}\right) e^{-i \mathrm{~K} z} J_{0}(k r) \mathrm{K} k d k,
$$




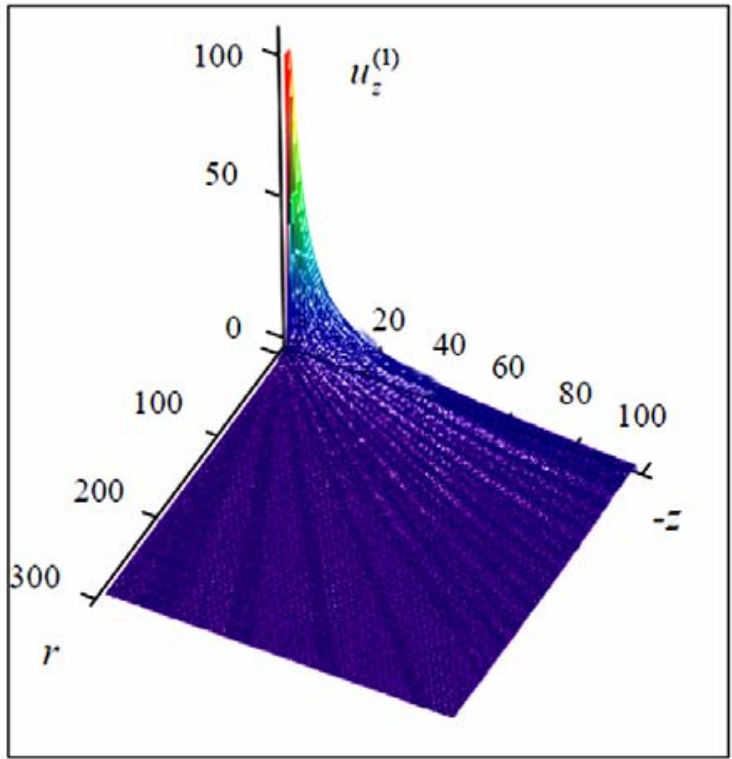

(a)

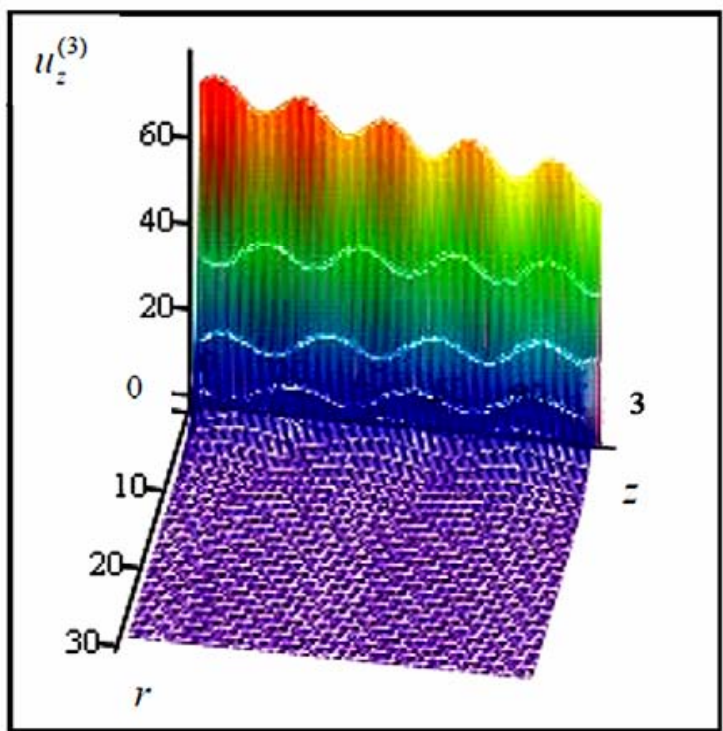

(c)

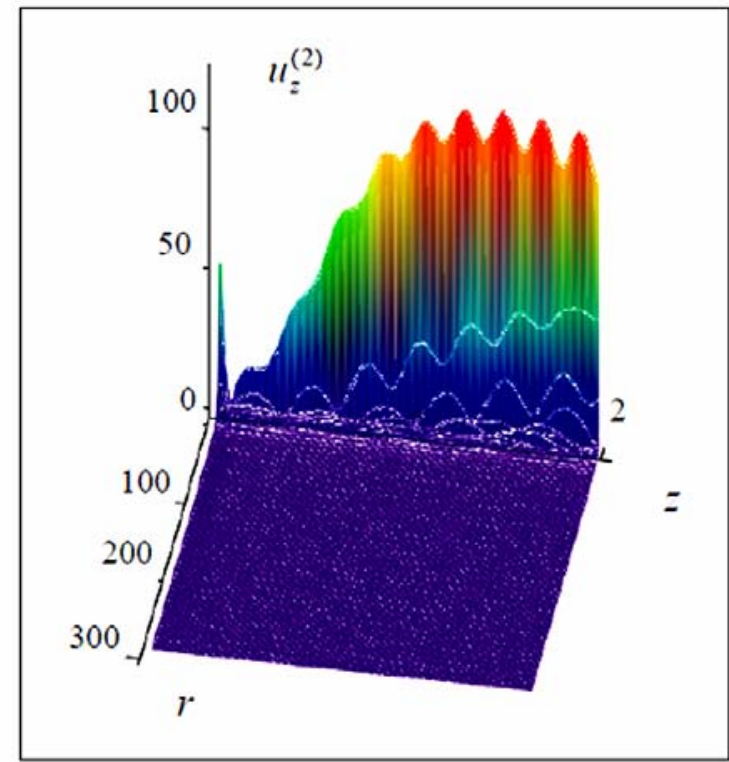

(b)

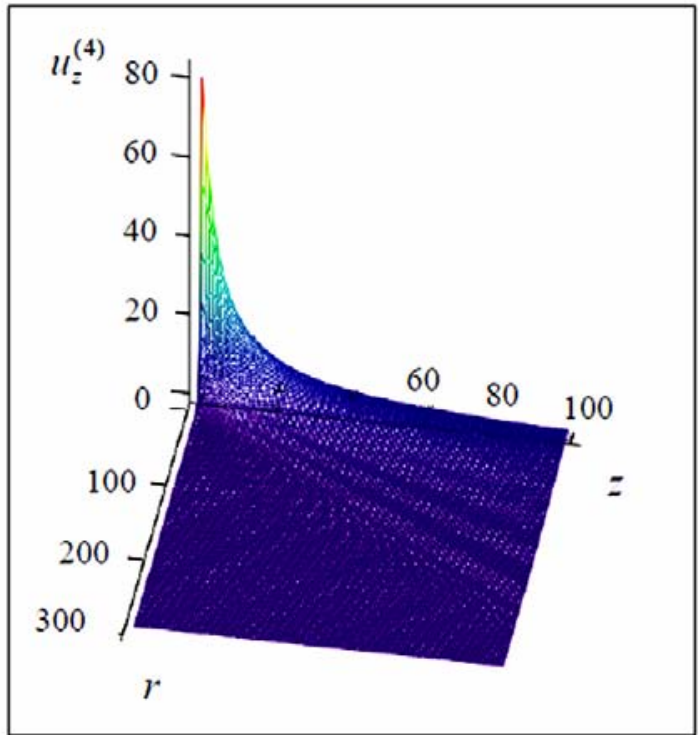

(d)

Figure 5. Fragments of the amplitude distribution pattern of the field $\left|u_{z}\right|$; relief over the coordinate plane $r-z:(a)-\operatorname{Re}-$ gion 1; (b) - Region 2; (c) - Region 3, and (d) - Region 4. $c^{\prime}=1.1, \omega h / C=9.1 \pi, \omega H / c=5 \pi$

$$
u_{z}^{(4)}=\frac{i F_{0}}{2 \pi \rho_{1} \omega^{2}} \int_{0}^{\infty} \frac{e^{i \mathrm{~K}(z-H)}}{2 \cos (\mathrm{KH})-i\left(\frac{\rho_{2}}{\rho_{1}}+\frac{\rho_{1}}{\rho_{2}}\right) \sin (\mathrm{KH})} J_{0}(k r) \mathrm{K} k d k
$$

These formulae are employed to carry out numerical calculation and analysis of the near acoustic field structure for different density contrasts in the layer and in the
Vicinity $\rho_{2} / \rho_{1}>1, \rho_{2} / \rho_{1}<1$, enabling one to determine the influence of variations in the ratio of the densities in inhomogeneity and in its vicinity. Figures 6 and 7 


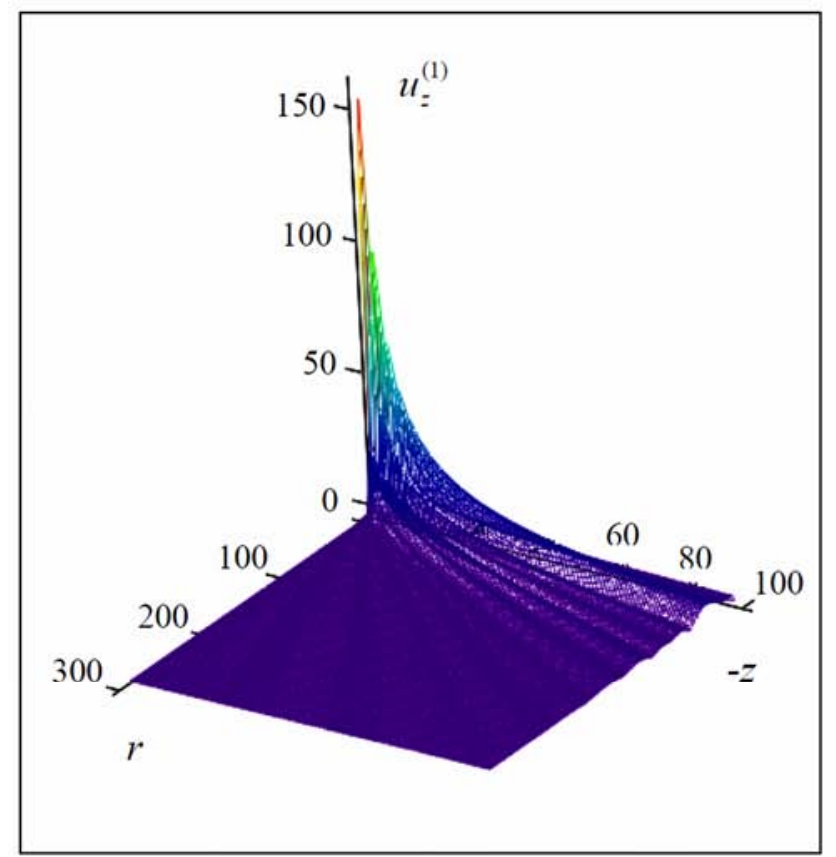

(a)

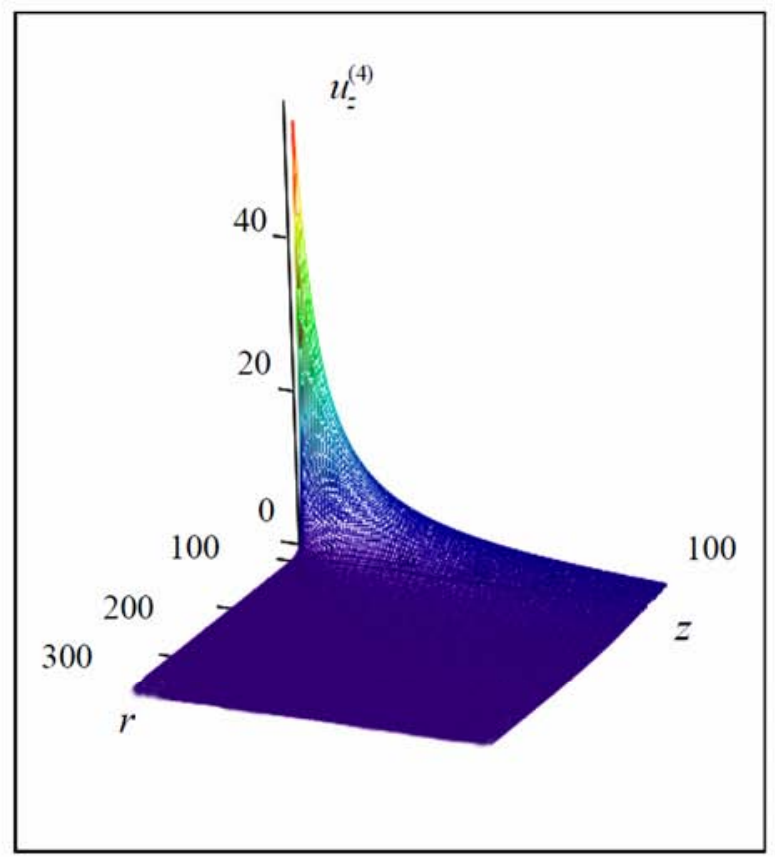

(b)

Figure 6. Fragments of the amplitude distribution pattern of the field $\left|u_{z}\right|: \omega h / C=10 \pi, \omega H / C=5 \pi, \rho_{2} / \rho_{1}=0.9 ;$ (a) - Region 1; (b) - Region 4

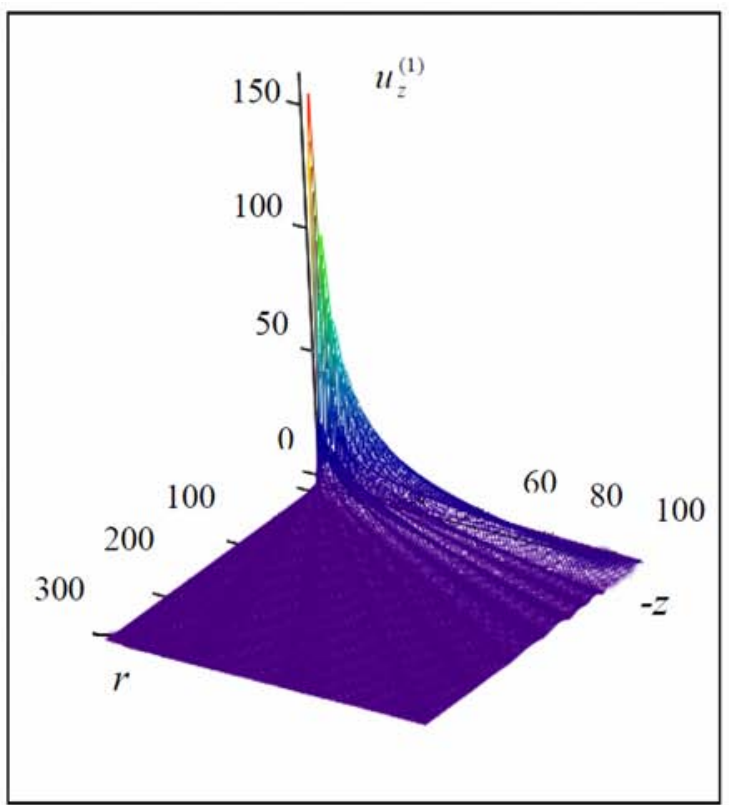

(a)

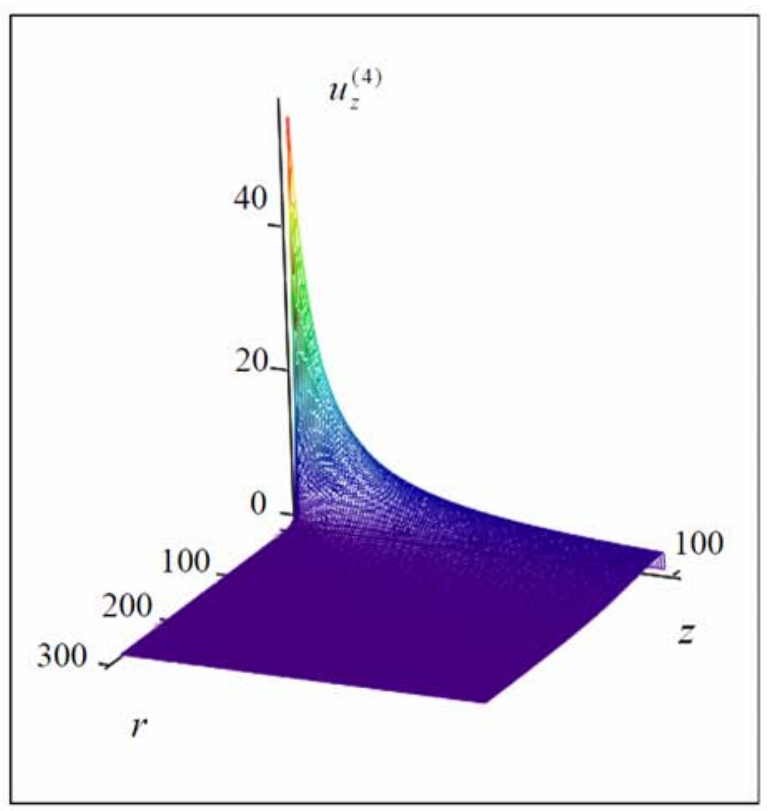

(b)

Figure 7. Fragments of the amplitude distribution pattern of the field $\left|u_{z}\right|: \omega h / C=10 \pi, \omega H / C=5 \pi, \rho_{2} / \rho_{1}=1.1 ;(a)$ - Region 1; (b) - Region 4.

deal with fragments of relief above the plane $r, Z$ in regions 1 and 4, which are calculated by Formulae (8) and (9) using the same values of the density ratio as in the velocity ratio calculations, i.e., $\rho_{2} / \rho_{1}=0.9, \quad \rho_{2} / \rho_{1}$ $=1.1$. These fragments are much similar to those considered above; the same picture is observed in interme- 
diate regions 2 and 3, thus neither appropriate fragments are shown nor are calculation formulas for these regions. It is seen in Figure 6(a) that if the media differ in density $\left(\rho_{2} / \rho_{1}=0.9\right.$ ), the distribution of the backscattered acoustic field is characterized (as in the previous case) by amplitude decrease and fan -shaped relief but of lower angular periodicity than that in Figure 4(a). The level of the field transmitted through the layer (see Figure 6(b)) decreases at larger distances from the external boundary but angular periodicity of amplitude values is absent. For a higher density contrast, i.e., for $\rho_{2} / \rho_{1}=1.1$ (see

Figures $\mathbf{7 ( a )}$ and $\mathbf{7 ( b )}$ ), the amplitude distribution patterns in reflected waves and waves transmitted through the layer remain practically invariable, which indicates a weak influence of the density contrast variation on the near acoustic field configuration in the layer - vicinity structure, as distinct from the previously considered velocity jump.

Generally, when comparing differentiation of media in density and velocity inside and outside the layer, we come to the conclusion that the sound speed jump in homogeneity causes a more pronounced variation of the near field configuration; thus the search system sensitivity to variation of this parameter is higher than the sensitivity to density contrast variation. This is the main distinction of these cases, which should be taken into account in the search for inhomogeneities and can be considered as one of the diagnostic properties enabling one to differentiate "inhomogeneities in density" and "inhomogeneities in velocity".

Therefore, the obtained fragments of the near acoustic field of a dipole harmonic source operating close to a layer inclusion yield the entire field pattern in principally different cases of velocity and density contrasts inside and outside inhomogeneity. The revealed peculiarities provide qualitative information on their applicability as informative attributes in the search for inhomogeneity. The distance from the source to the nearest (face) boundary of the layer, the thickness of the layer, and hence the sound speed (density) in region 3, i.e., in the zone occupied by probed inhomogeneity, is determined by the field configurations in regions 1,2 , and 4 . Thus remote reading of the inhomogeneity parameters in the harmonic oscillation mode requires "reflection" and "transmittance" probing. More detailed numerical simulation of near fields will provide quantitative data on the relation of inhomogeneity contrast against the vicinity to the spatial structures of these fields in the parameters of density and sound speed. Finally, it can be noted that the illustrations confirm our statements only qualitatively; the problem of frequency choice optimization required for practical acoustic probing is not considered.

\section{Transverse SH-Wave Field Generated in an Infinite Medium by an Extended Oscillating Power Source Close to a Layer Inclusion (Two-Dimensional Problem)}

The considered vibration source $Z_{0} \vec{z}^{0} \delta(x) \delta(y) e^{-i \omega t}$ is the "force oscillating with the frequency $\omega$ " (the force vector is parallel to the unit vector $\vec{Z}^{0}$ and has the amplitude $Z_{0}$; the factor $e^{-i \omega t}$ is omitted as previously) is uniformly distributed along the axis $z$ due to the two-dimensional approximation used in the analysis (see Figure 8). The source is omnidirectional relatively to radiated $S H$-waves, i.e., in the plane $x, y$ oriented normally to the $\vec{Z}^{0}$ direction. Thus besides comparing acoustic and vibroseismic cases in this analysis, it is possible to study the influence of the source directivity on the near field characteristics. As in the previous case, the layer occupies the spatial region $-\infty<x<\infty, h \leq$ $y \leq h+H,-\infty<z<\infty$. The vicinity is characterized by the transverse wave velocity $C_{t}$ and differs from the analogous value $c_{t}$ inside the layer; both media have the same density $\rho$.

It is shown in References [4-6] that to describe the wave displacements $u_{z}$ in the two-dimensional problem, it is sufficient to introduce one component of the vector potential $\psi_{x}$ satisfying the homogeneous wave equation:

$$
\begin{aligned}
& \Delta \psi_{x}^{(1,2,4)}-\frac{1}{C_{t}^{2}} \frac{\partial^{2} \psi_{x}^{(1,2,4)}}{\partial t^{2}}=0, \\
& \Delta \psi_{x}^{(3)}-\frac{1}{c_{t}^{2}} \frac{\partial^{2} \psi_{x}^{(3)}}{\partial t^{2}}=0 .
\end{aligned}
$$

By analogy with the previous case of a dipole source in a scalar medium, the entire space is divided into four especially distinguished regions (see Figure 8); in each region the value $\psi_{x}$ is represented as the Fourier expansion, i.e., by the following expressions:

$$
\begin{gathered}
\psi_{x}^{(1)}=\int_{-\infty}^{\infty} A(k) e^{-i K_{t} y+i k x} d k, \quad y<0, \\
\psi_{x}^{(2)}=\int_{-\infty}^{\infty}\left(B(k) e^{i K_{t} y}+C(k) e^{-i K_{t} y}\right) e^{i k x} d k \\
0<y<h \\
\psi_{x}^{(3)}=\int_{-\infty}^{\infty}\left(a(k) e^{i \kappa_{t} y}+b(k) e^{-i \kappa_{t} y}\right) e^{i k x} d k
\end{gathered}
$$




$$
\begin{gathered}
h<y<h+H \\
\psi_{x}^{(4)}=\int_{-\infty}^{\infty} D(k) e^{i K_{t} y+i k x} d k, h+H<y<\infty
\end{gathered}
$$

where $\mathrm{K}_{t}=\sqrt{\omega^{2} / C_{t}^{2}-k^{2}}, \kappa_{t}=\sqrt{\omega^{2} / c_{t}^{2}-k^{2}}$, $C_{t}$ and $c_{t}$ are the shear wave velocities in the vicinity and inside the layer and $k$ is the integration variable.

The oscillation displacements $u_{z}$ and significant stresses in the considered waves $\sigma_{y z}$ are expressed through $\psi_{x}$ using differential operations:

$$
u_{z}^{(1.4)}=-\partial \psi_{x}^{(1.4)} / \partial y
$$

$$
\sigma_{y z}^{(1,2,4)}=\rho C_{t}^{2} \partial u_{z}^{(1,2,4)} / \partial y, \sigma_{y z}^{(3)}=\rho c_{t}^{2} \partial u_{z}^{(3)} / \partial y,
$$

where $\rho$ is the density of the medium; the source operation is described by one of the conditions for $y=0$ instead of appropriate expressions in the right-hand side of Equation (10). The boundary condition is:

$$
\sigma_{y z}^{(2)}(y=0)-\sigma_{y z}^{(1)}(y=0)=Z_{0} \delta(x)
$$

The unknown coefficients $A(k), B(k), C(k), a(k)$, $b(k), D(k)$ are found by matching the indicated shift components and strains at the boundaries of the four distinguished regions. Omitting intermediate calculations, we write down the resultant expressions for the wave displacements:

$$
\begin{aligned}
& u_{z}^{(1)}=\frac{-i Z_{0}}{4 \pi \rho C_{t}^{2}} \int_{-\infty}^{\infty}\left(1+\frac{2 i\left(\frac{c_{t}^{4} \kappa_{t}^{2}}{C_{t}^{4} \mathrm{~K}_{t}^{2}}-1\right) \sin \left(\kappa_{t} H\right)}{\left(\left(\frac{c_{t}^{2} \kappa_{t}}{C_{t}^{2} \mathrm{~K}_{t}}+1\right)^{2} e^{-i \kappa_{t} H}-\left(\frac{c_{t}^{2} \kappa_{t}}{C_{t}^{2} \mathrm{~K}_{t}}-1\right)^{2} e^{i \kappa_{t} H}\right)} e^{i 2 K_{t} h}\right) \frac{e^{-i K_{t} y+i k x}}{\mathrm{~K}_{t}} d k \\
& u_{z}^{(2)}=\frac{-i Z_{0}}{4 \pi \rho C_{t}^{2}} \int_{-\infty}^{\infty}\left(e^{i K_{t} y}+\frac{2 i\left(\frac{c_{t}^{4} \kappa_{t}^{2}}{C_{t}^{4} \mathrm{~K}_{t}^{2}}-1\right) \sin \left(\kappa_{t} H\right) e^{-i \mathrm{~K}_{t}(y-2 h)}}{\left(\left(\frac{c_{t}^{2} \kappa_{t}}{C_{t}^{2} \mathrm{~K}_{t}}+1\right)^{2} e^{-i \kappa_{t} H}-\left(\frac{C_{t}^{2} \kappa_{t}}{C_{t}^{2} \mathrm{~K}_{t}}-1\right)^{2} e^{i \kappa_{t} H}\right)} \frac{e^{i k x}}{\mathrm{~K}_{t}} d k,\right. \\
& u_{z}^{(3)}=\frac{-i Z_{0}}{2 \pi \rho C_{t}^{2}} \int_{-\infty}^{\infty} \frac{\left(\left(\frac{c_{t}^{2} \kappa_{t}}{C_{t}^{2} \mathrm{~K}_{t}}+1\right) e^{i \kappa_{t}(y-H-h)}+\left(\frac{c_{t}^{2} \kappa_{t}}{C_{t}^{2} \mathrm{~K}_{t}}-1\right) e^{-i \kappa_{t}(y-H-h)}\right)}{\left(\left(\frac{c_{t}^{2} \kappa_{t}}{C_{t}^{2} \mathrm{~K}_{t}}+1\right)^{2} e^{-i \kappa_{t} H}-\left(\frac{c_{t}^{2} \kappa_{t}}{C_{t}^{2} \mathrm{~K}_{t}}-1\right)^{2} e^{i \kappa_{t} H}\right)} \frac{e^{i K_{t} h+i k x}}{\mathrm{~K}_{t}} d k, \\
& u_{z}^{(4)}=\frac{-i Z_{0} c_{t}^{2}}{\pi \rho C_{t}^{4}} \int_{-\infty}^{\infty} \frac{\kappa_{t} e^{i \mathrm{~K}_{t}(y-H)+i k x}}{\mathrm{~K}_{t}^{2}\left(\left(\frac{C_{t}^{2} \kappa_{t}}{C_{t}^{2} \mathrm{~K}_{t}}+1\right)^{2} e^{-i \kappa_{t} H}-\left(\frac{c_{t}^{2} \kappa_{t}}{C_{t}^{2} \mathrm{~K}_{t}}-1\right)^{2} e^{i \kappa_{t} H}\right)} d k
\end{aligned}
$$

It follows from (15) that similarly to the previous case with an acoustic dipole, the field $u_{z}^{(4)}$ does not depend on the parameter $h$, i.e., the distance between the source and the nearest layer boundary, which is the consequence of the unlimited scale of inhomogeneity along the coordinate $X$ and the absence of absorption in the medium. The obtained expressions are used in the numerical calculation enabling one (by means of computer graphics) to visualize the spatial distribution of wave amplitudes at the distances of up to several tens of wavelengths from the source for the layer thickness of the order of or smaller than the wavelength and to analyze the peculiari- ties of this spatial distribution. Specifically, these expressions are used to make calculations and obtain patterns of the wave displacement field (in the isometric projection) for the relative distance from the source and the layer thickness assigned in the dimensionless form: $\omega h / C_{t}=9 \pi, \omega H / c_{t}=5 \pi$. As previously, the calculations are carried out for two velocity jumps $c_{t} / C_{t}=0.9$ and $c_{t} / C_{t}=1.1$. As the integral expressions, each of the four fragments of the field pattern corresponds to its spatial region; the amplitude distribution is shown as a relief rising above the plane $x, y$.

Figures 9(a), (b), (c), and (d) should be considered in 


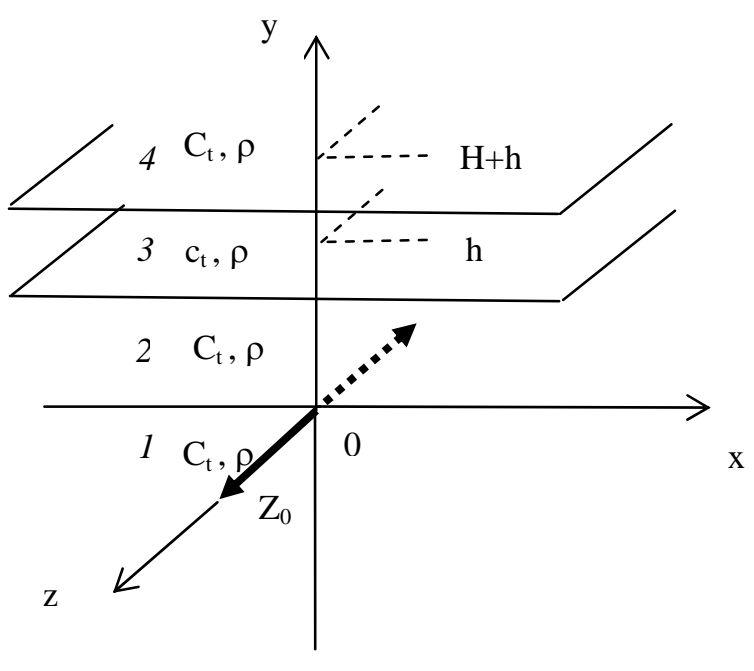

Figure 8. Mutual arrangement of the source and the layer scattering of shear $\mathrm{SH}$-waves

the following sequence: Figure 9(a) - region 1, Figure 9(b) - region 2, etc., while for obtaining the entire field pattern in all the regions all the fragments should be joined. Since Figure 9(a) should be considered turned in the opposite direction along the transverse coordinate $y$, the corresponding axis in it is denoted $-y$. It follows from the given pattern that in region 1 at larger distances from the source, besides a decrease of the field $\left|u_{z}^{(1)}\right|$ we observe periodic sequences of maxima occupying fanshaped angular sectors, which pass into directional lobes in the far field. Note that the scale of the longitudinal coordinate $x$ is the same in all the figures, while the scale of the transverse coordinate $y$ in Figures 9(b) and 9(c) is two orders smaller than that in Figures 9(a) and 9(d), i.e., the unit length in the transverse direction $y$ in Figures

9(b) and 9(c) is 100 times larger than that in Figures 9(a) and 9(d). Taking into account the scale difference it can be concluded that at larger distances from the source and approach to the layer boundary in the second region, the amplitude of the field $\left|u_{z}^{(2)}\right|$ decreases even more abruptly than that in Figure 9(a). The amplitude of the field penetrating into the layer is maximum in the region opposite to the source, decreases abruptly when escaping along the coordinate $x$ (symmetrically on both sides), and oscillates along the coordinate $y$. It is seen in Figure 9(c) that waveguide conditions for excitation and propagation of several first modes of $\mathrm{SH}$-wave occur inside the layer. The field $\left|u_{z}^{(4)}\right|$ outside the layer (see Figure 9(d)) also decreases rapidly and the amplitude distribution in region 4 differs considerably from the analogous one in region 1. A similar situation is considered in the first section for a dipole source in the scalar acoustic problem.

To gain a better understanding of wave reflections occurred in near-field probing in the near region of the source, it is expedient to consider antiwaveguide propagation for an inverse jump of $\mathrm{SH}$-wave velocities inside the layer and in the vicinity, which equals, for example, $c_{t} / C_{t}=1.1$.

Figures 10(a), (b), (c), and (d) exhibit analogous fragments of the spatial field distribution in the same format and in the same spatial regions as in the figure considered above. Comparison of the amplitude distributions with the analogous ones of the previous case (Figure 9) shows that the spatial dependence can be either the same or slightly different. The near field in the reflection region (Figure 10(a) - region 1) has practically the same structure as in the previous case. The configurations of the amplitude distributions in region 2 (Figures 10(b)) in those cases are also similar. The field configuration in the layer (Figures 10(c)), i.e., in region 3 , differs by the absence of periodic structure indicating the excitation of $\mathrm{SH}$-wave modes, in spite of the presence of a crest with undulatory amplitude modulation also typical of the previous case. In region 4 immediately outside the layer limits (see Figures 10(d)), an increased-amplitude angular sector forms being similar to that in the scalar acoustic problem.

The revealed peculiarities differentiating the structures of the near fields traveling in opposite directions from the layer in waveguide and antiwaveguide cases demonstrate the possibility of remote diagnostics of elasticity jump in the media occupying the internal and external regions of the layer and enable one to accept them as informative diagnostic attributes applicable, specifically, for solving problems of remote diagnostics and medium structure retrieval. Hence the previously formulated statement on the necessity of reflected and transmitted wave recording for diagnostics of inhomogeneity in its near-field probing, which is similar to reflection and transmittance location, is valid. Generally, similar results on peculiarities of wave reflection and transmittance through a layer several wavelengths thick are typical of the scalar acoustic problem and the problem with a source exciting $\mathrm{SH}$-waves in an elastic medium.

\section{Conclusions}

Numerical simulation of near-field probing of inhomogeneity (layer inclusion) in acoustic and seismic media is carried out, which has confirmed its applicability with the use of near acoustic and elastic fields of harmonic sources and recording of waves reflected by in homogeneity and transmitted through it. The study is based on the analysis of the visual pattern of the spatial amplitude distribution in near and far wave fields calculated by the formulae derived in this paper. The simultaneously considered angular characteristics of the far 


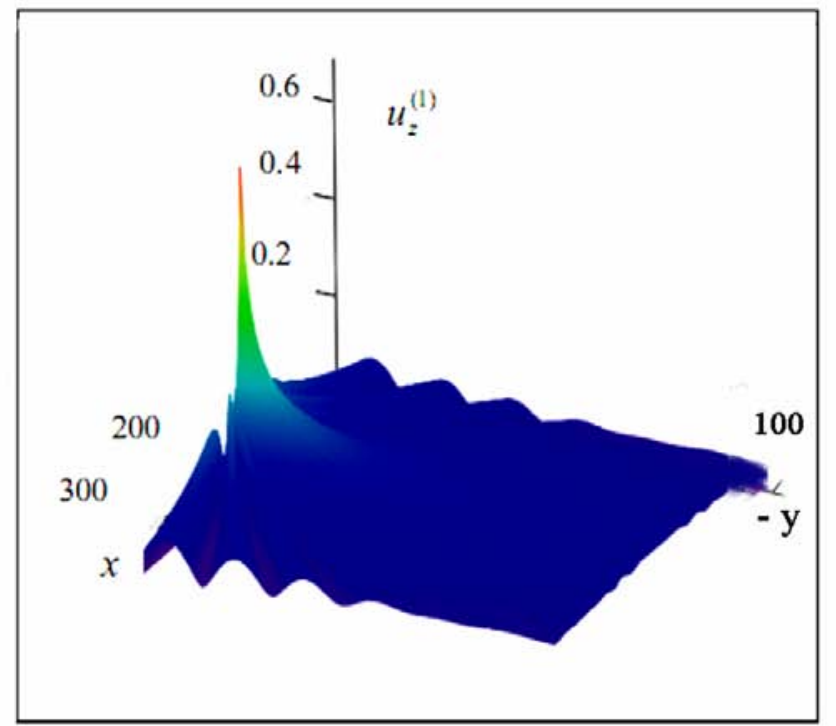

(a)

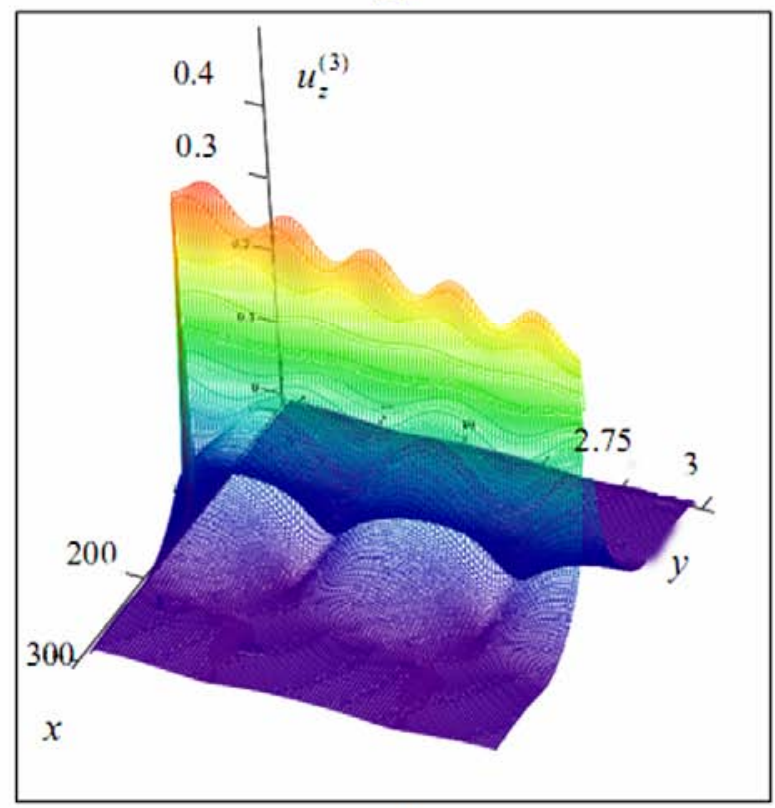

(c)

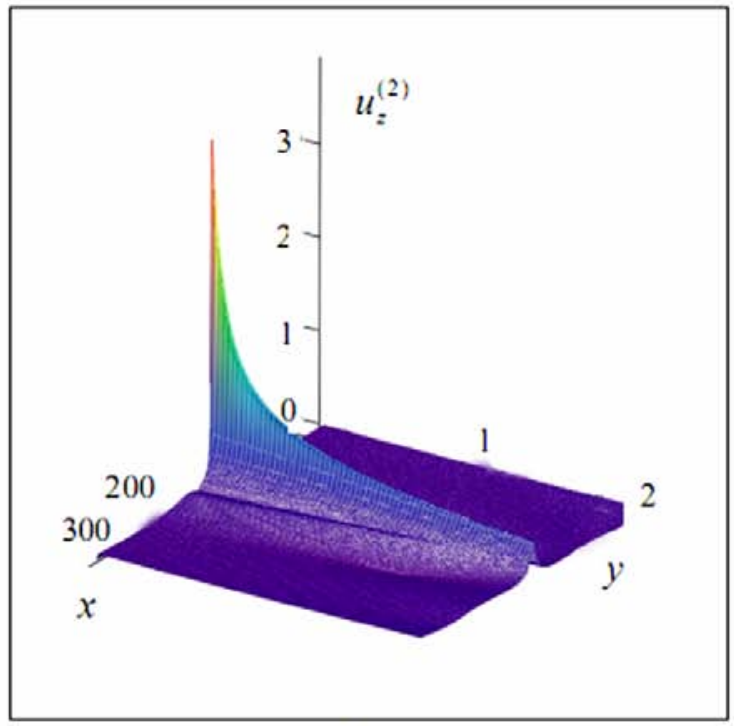

(b)

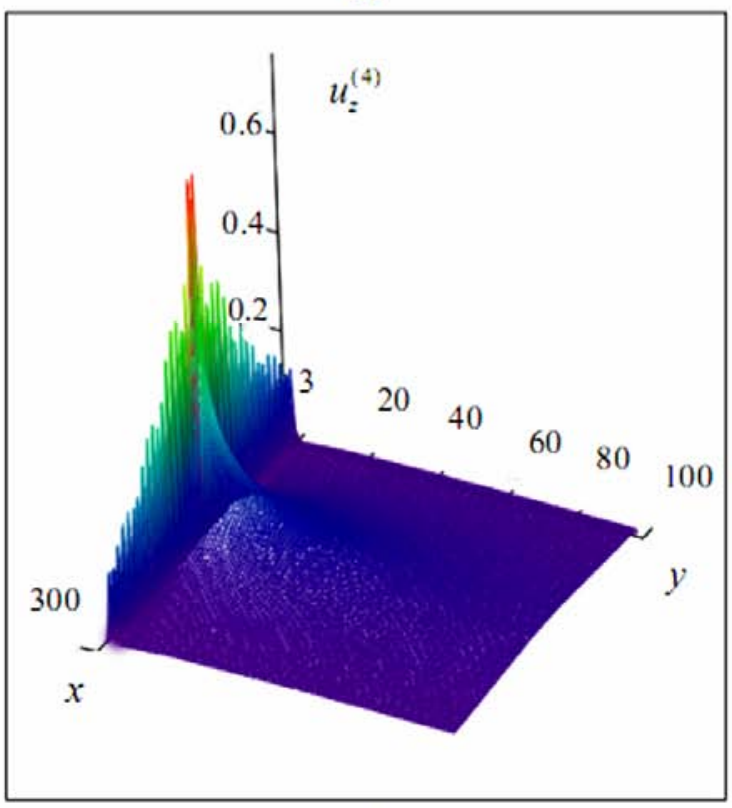

(d)

Figure 9. Fragments of the spatial distribution of wave amplitudes of an oscillating source for $c_{t} / C_{t}=0.9, \omega h / C_{t}=11.1 \pi$, $\omega H / c_{t}=5 \pi$ : (a) - Region 1; (b) - Region 2; (c) - Region 3; (d) - Region 4

acoustic field do not contradict the revealed peculiarities of the near field of elementary oscillation sources operating close to inhomogeneity. The employed values of inhomogeneity contrast characterize the relation of densities and sound speeds in the layer and ambient acoustic medium. To predict the distance from the source to the layer inclusion and to estimate its thickness, the qualitative character of the dependence $\omega H / c, \omega h / C$ $\left(\omega H / c_{t}, \omega h / C_{t}\right)$ should be studied. Complete investigations require numerical simulation of a number of definite values of the mentioned parameters in addition to the given calculations. At the same time, the near field peculiarities found in this paper (even in a limited volume of simulation data) are useful for optimal arrangement of sources and recording receivers in design of experiments on seismic exploration of productive stratum in massif, characterized by an abrupt decrease of $\mathrm{SH}$-wave velocity. 


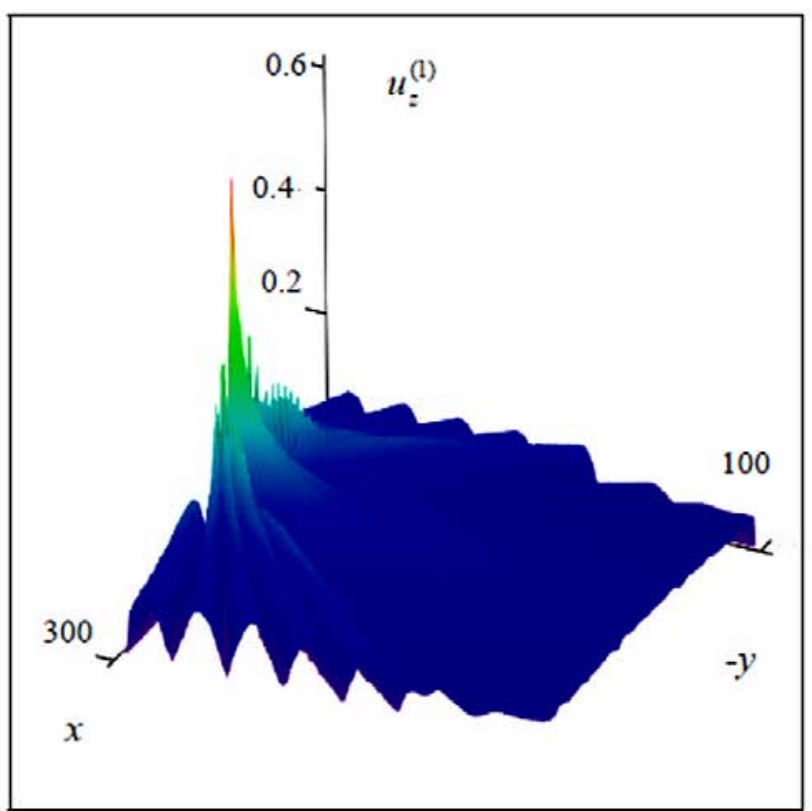

(a)

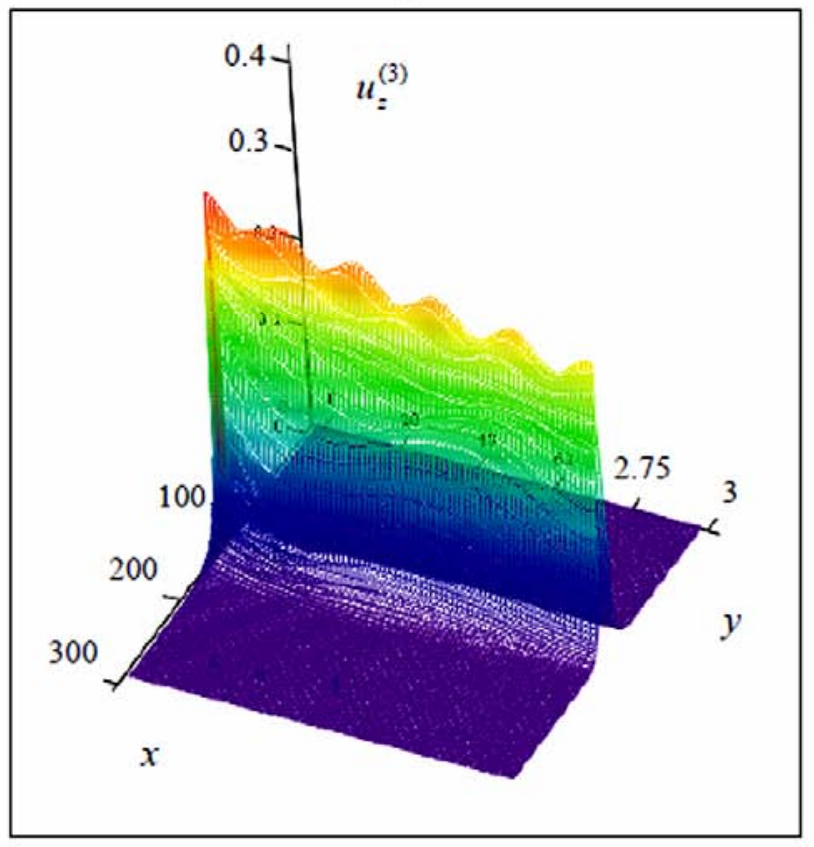

(c)

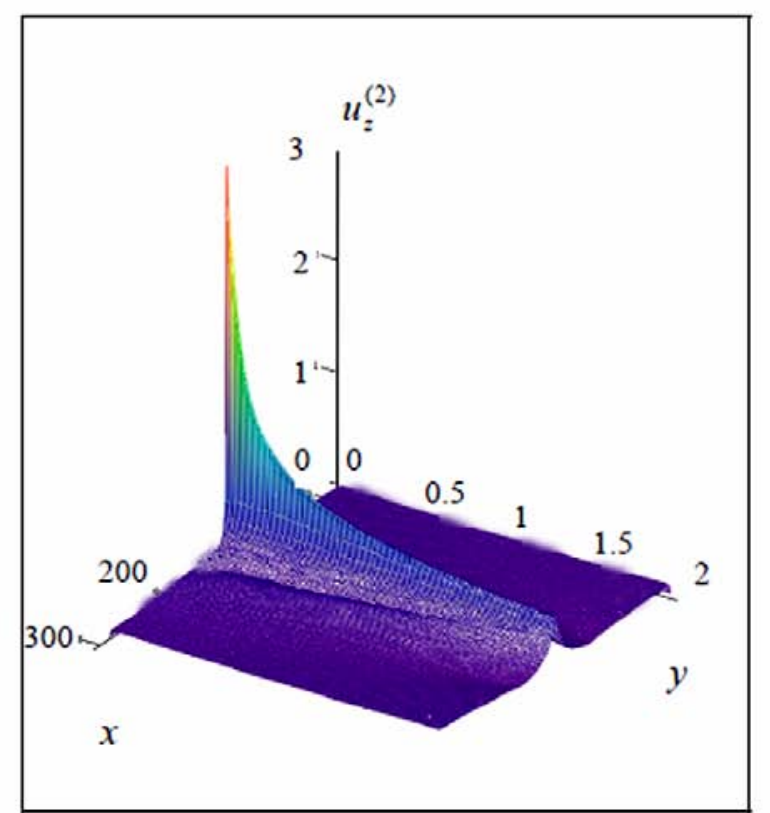

(b)

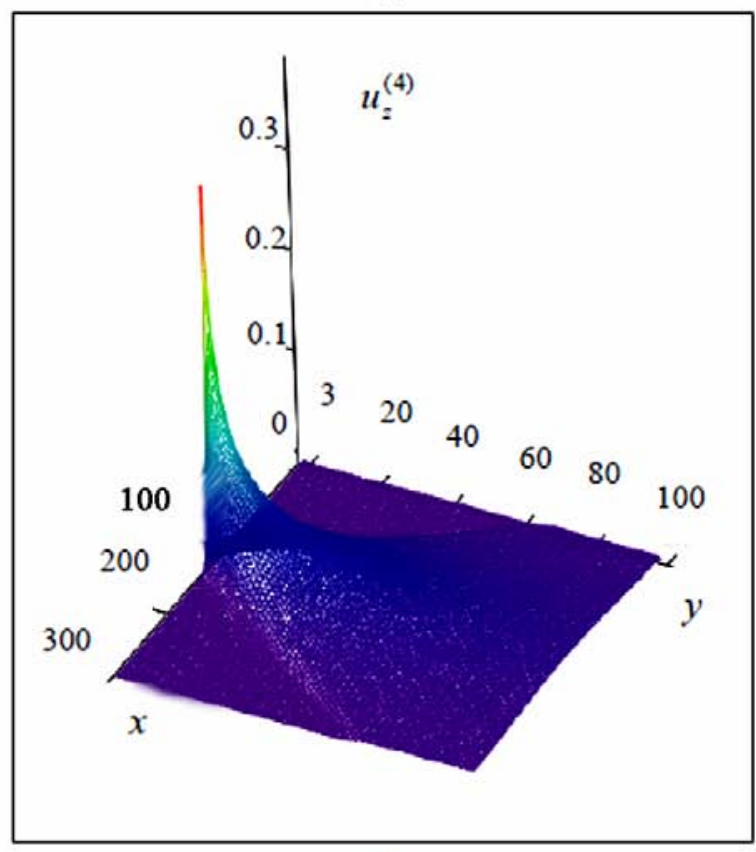

(d)

Figure 10. Fragments of the spatial distribution of the wave field for $c_{t} / C_{t}=1.1, \omega \mathrm{h} / C_{t}=9.1 \pi, \omega H / c_{t}=5 \pi:(\mathrm{a})-\mathrm{Region} 1$; (b) - Region 2; (c) - Region 3; (d) - Region 4

The structures of the near fields of a vibration source, which are backscattered or transmitted through inhomogeneity, should be considered as a set of informative basic characteristics indirectly indicating the presence of a stratum with deposit. Shelf investigations of sea bottom sediments containing gas condensate layers can be similar to the search for hydrocarbon accumulation in geo- logic environment on land territories. In some cases, the search for inhomogeneities using harmonic oscillation sources can precede pulse location and determine only tentative information or boundary contours. In other cases, it is expedient to employ near-field probing using harmonic sources to increase reliability of pulse echosounding of geological structures or prediction accuracy 
of their characteristics in remote diagnostics [9].

\section{REFERENCES}

[1] A. M. Derzhavin, O. V. Kudryavtsev and A. G. Semenov, "On Peculiarities of Numerical Simulation of a Vector Wave Field of a Low-Frequency Acoustic Source in the Ocean Medium,” Akusticheskii Zhurnal, Vol. 46, No. 4, 2000, pp. 480-489.

[2] L. A. Bespalov, A. M. Derzhavin and O. V. Kudryavtsev, "On Simulation of a Seismoacoustic Field of a LowFrequency Source for Variation in the Structure of Ocean Bottom Thickness,” Akusticheskii Zhurnal, Vol. 45, No.1, 1999, pp. 25-37.

[3] L. M. Brekhovskikh, "Waves in Layered Media,” Nauka, Moscow, 1973, p. 343.

[4] Y. M. Zaslavsky and V. Y. Zaslavsky, "Analysis of a Vibroacoustic Field in an Extended Layer and a LayerSemispace Structure," Izvestiya Vysshikh Uchebnykh Za- vedenii, Radiofizika, Vol. 7, No. 2, 2009, pp. 151-163.

[5] Y. M. Zaslavsky and V. Y. Zaslavsky, “Analysis of an Acoustic Field Excited by a Vibration Source in a Layer and its Vicinity,” Akusticheskii Zhurnal, Vol. 55, No. 6, 2009, pp. 845-852.

[6] Y. M. Zaslavsky and V. Y. Zaslavsky, "Transverse Waves Excited by a Variable Power Source in a Layer and its Vicinity," NNGU Vestnik, Radiofizika, 2009, No. 5, pp. 61-68.

[7] Y. M. Zaslavsky, B. V. Kerzhakov and V. V. Kulinich, "Vertical Seismic Profiling on a Sea Shelf," Akusticheskii Zhurnal, Vol. 54, No. 3, 2008, pp. 483-490.

[8] Y. M. Zaslavsky, B. V. Kerzhakov and V. V. Kulinich, "Simulation of Wave Radiation and Phased Antenna Reception on the Ocean Shelf,” Akusticheskii Zhurnal, Vol. 53, No. 2, 2007, pp. 264-273.

[9] J. L. Arroyo, P. Breton, H. Dijkerman, et al. "Superior Seismic Data from the Borehole,” Oilfield Review, Vol. 15 , No. 1, 2003, pp. 1-23. 